\title{
The Different Muscle Activation of Upper Extremity and Core Muscle by the Changes of Leg Support Surface during Push-up Exercise
}

\author{
Sun-Uk Kim', Seong-Bin Kim', Sang-Seok Yeo² \\ 'Department of Industrial Engineering, College of Convergence Technology, Dankook University; ${ }^{2}$ Department of Physical Therapy, College of Health \\ Sciences, Dankook University, Cheonan, Korea
}

Purpose: This study was designed to evaluate the effects of the different condition of leg support surface on the upper extremity and core muscle activity during the push-up exercise.

Methods: Fifteen healthy subjects that were practicable push-ups were recruited in this study. Subjects were instructed the push-up exercise in the different condition of the leg support surface. Each condition of support surface was set to the high and lower, and the unstable and stable condition. Muscle activation was measured by using the surface electromyography (EMG), and recorded from the triceps brachii, serratus anterior, latissimus dorsi, rectus abdominis, abdominal external oblique, and erector spinea muscle.

Results: In the results of experiments, there was no significant difference of muscle activation in upper extremity between the high unstable and high stable support surface. By contrast, muscle activation of the rectus abdominis and abdominal external oblique was significantly higher in the low unstable support surface, compared with those of the low stable support surface. It is well known that the core muscle was important to stabilization of trunk stability.

Conclusion: This result demonstrates that the low and unstable support surface for the lower extremity was suited for training of core muscle for trunk stabilization during the push-up exercise.

Keywords: Core muscle, Push-up exercise, Electromyography

\section{INTRODUCTION}

The push-up exercise is one of an easy and popular exercise that can be widely used in general exercise programs in order to strengthen the upper extremity. In addition, it is evaluated as an effective exercise method that can contribute to a healthy recovery by preventing shoulder damage and improving the muscle strength near the joints after injury. ${ }^{1-3}$ The push-up exercise is known to be a representative closed kinetic chain exercise that targets the pectoralis major and triceps brachii, as well as the scapular stabilizing muscles. As such, the push-up exercise can activate co-contraction of various muscles, which can improve dynamic stability of joints and upright posture maintenance. Moreover, it provides facilitation of proprioceptive feedback by stimulating afferent receptors around joints, and en-

Received May 20, 2016 Revised Jun 13, 2016

Accepted Jun 14, 2016

Corresponding author Sang-Seok Yeo

E-mail yeopt@dankook.ac.kr hancing muscle strength and endurance. ${ }^{2,4}$

The push-up exercise can be performed either on a stable support surface such as floor or wall, or on an unstable support surface, such as a Swiss ball or Variations in the push-up exercise may change muscular activation pattern. Many studies have reported that muscle activity of upper extremity changes when they compared the unstable push-up exercise with the stable push-ups. ${ }^{5-7}$ The shoulder stabilizer muscles, such as the serratus anterior, levator scapulae, rhomboid, upper and lower trapezius, were increased during the push-up exercise on an unstable surface. Moreover, there are other muscle groups, such as anterior shoulder and core muscle, are involved and it effectively improves balance by stimulating muscle proprioceptors. In addition, many previous studies reported that experiments under various environmental conditions were con-

Copylight (C2016 The Korea Society of Physical Therapy

This is an Open Access article distribute under the terms of the Creative Commons Attribution Non-commercial License (Http:// creativecommons.org/license/by-nc/4.o.) which permits unrestricted non-commercial use, distribution, and reproduction in any medium, provided the original work is properly cited. 
ducted to examine the therapeutic effects of push-up exercise..$^{7-9}$ It is known that there are differences in the muscle activities of the upper extremity muscles with regard to the shoulder width, hand position, slope and height of a support surface during push-up exercise. ${ }^{10-12}$ However, scores of the recent studies on exercise therapy, including push-up exercise, have reported on the effects of exercises on an unstable surface to indicate that such exercises improve the strength of core muscles that is mainly needed to maintain a posture. ${ }^{13,14}$ The core muscles which are deep muscles in the trunk are largely divided into abdominal muscles and lower-back muscles. The abdominal muscles include the rectus abdominis muscle, the abdominal external oblique muscle, the abdominal internal oblique muscle, and the abdominal transversus muscle. ${ }^{15}$ The main muscles of lower-back muscles are the multifidus and erector spinea muscle. ${ }^{15,16}$

Sling exercises and Swiss ball exercises were used in the studies aimed at examining the effects of exercises on an unstable surface, and the effects of such exercises have been proven improve muscles activities in the trunk and shoulders through many studies. ${ }^{14}$ Nonetheless, push-up exercise on an unstable surface is focused on providing an unstable support surface of hands and is limited to measuring the patterns of change in muscle activities of the upper extremity muscles. Though there are some recent studies on push-up exercise, reporting on the patterns of change in muscle activities by making a support surface of the lower limbs unstable, they are limited to measuring and proving the patterns of change in the muscle activities of only the upper extremity muscles. ${ }^{17}$ Therefore, identification of different activation in upper extremity muscle and core muscle during push-up exercise on an unstable surface can provide useful information for clinicians and researchers in the therapeutic exercise.

The present study aims to investigate differences in muscle activities of the upper extremity muscles and the core muscles during push-up exercise on an unstable surface and a stable surface of the lower limbs.

\section{METHODS}

\section{Subjects}

For the purpose of the present study, 15 male college students who did not have the history of muscular illness to do push-ups were selected. After being informed of the purpose and method of the study, they were the ones who agreed to voluntary participation of the study.

\section{Experimental equipment}

Telemyo 2400T (Noraxon, USA) used in the experiment is a device that uses surface electromyograms (EMG) for measuring muscle activities during push-up exercise. Noraxon Myo Reserach Master Edition 1.08 XP Software was used in the experiment to collect and analyze the muscle activities. On measuring sites, circular type 2 Ag/AgCl electrodes (3M, USA) were used. Gym balls and rubber balls were used as unstable surfaces while flat height-adjustable benches were used as stable surfaces after adjusting their height similar to those of the gym ball and the rubber ball.

\section{Electromyography measurement}

To carry out the present study, hair on the skin where sensors would be attached was removed and its surface was cleaned with alcohol in order to minimize skin resistance. Electrodes were attached to the triceps brachii, the serratus anterior, the latissimus dorsi, the rectus abdominis muscle, the abdominal external oblique muscle and the erector spinea muscle of the study subjects in order to measure the muscle activities of their upper extremity muscles and core muscles. ${ }^{13,14,17}$ Active electrodes were attached to muscle bellies that show the clearest muscular contraction with maximum muscular contraction while the subjects were in the position for a Manual Muscle Test (MMT) of each muscle. ${ }^{10}$ In addition, amplified waves were filtered through a Bandpass filter of $10-350 \mathrm{~Hz}$ for signal processing. The mean value of root mean squares (RMS) was used for each muscle's activity.

\section{MVIC measurement}

Maximal Voluntary Isometric Contraction (MVIC) was performed and measured while the subjects were in the position for a MMT in order to normalize the value of EMG activity. ${ }^{11,18}$ The MVIC was measured for five seconds and for three seconds except the first and the last second, the mean EMG signal was used. The normalized value of muscle activities in the upper extremity muscles and the core muscles was computed after setting the MVIC value at $100 \%$ and dividing the value of each muscle's activity during push-up exercise by the MVIC value. 


\section{Experimental procedure}

The experiment was carried out after checking the signals of the attached electrodes. However, push-up exercises on an unstable surface and a stable surface were performed in a random order and the push-up movements were classified into arm-folding and arm-unfolding movements. The push-up exercise on a stable surface was performed with the hands at the shoulder width and the feet on stable support surfaces of $55 \mathrm{~cm}$ and $25 \mathrm{~cm}$ off the ground, and its mean value was calculated after repeating the exercise for three times. On the other hand, the push-up exercise on an unstable sur-

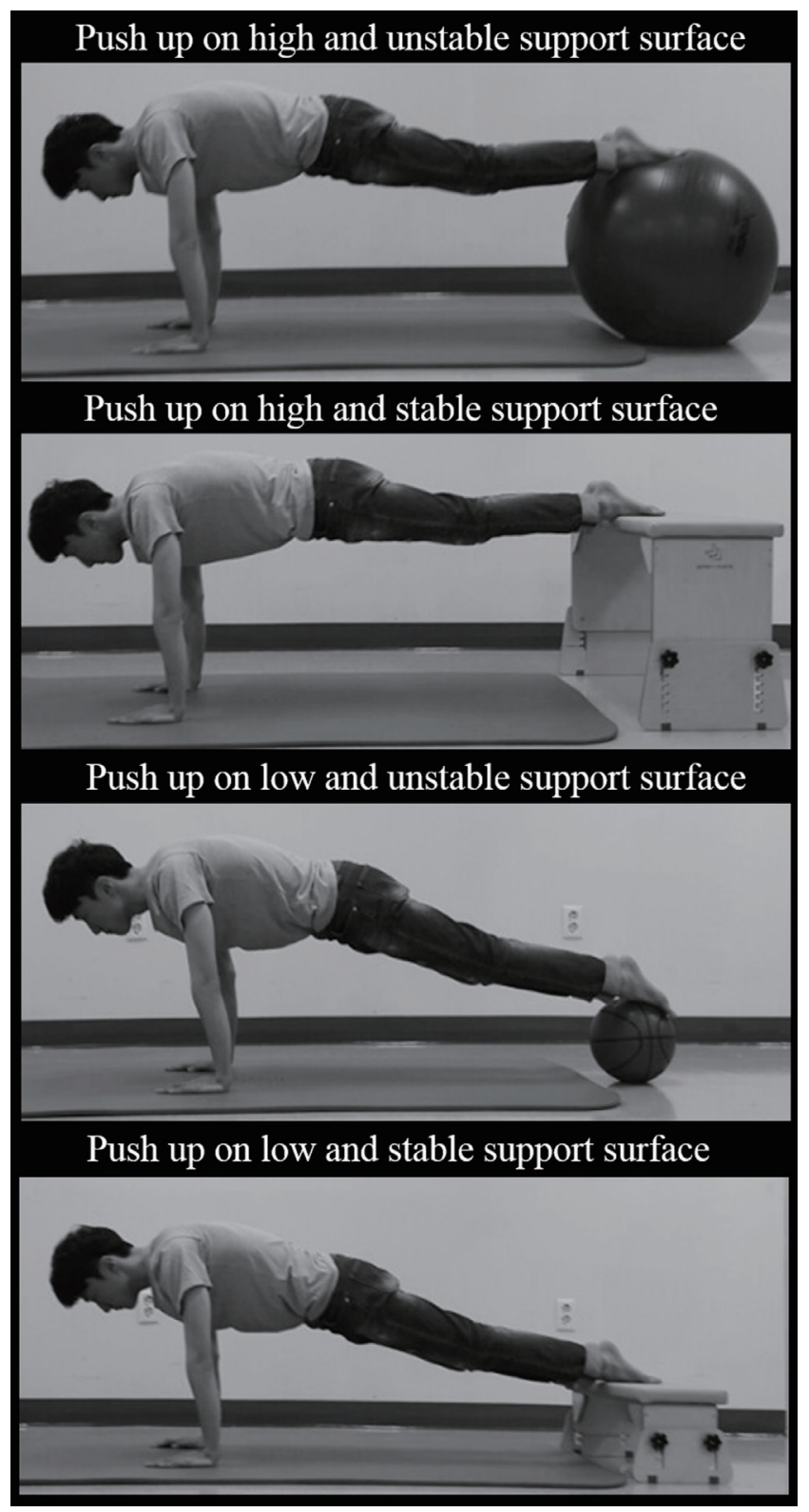

Figure 1. Different conditions of push up exercise. face was performed with the hands at the shoulder width just like in the exercise on a stable surface, however the feet on rubber balls of $55 \mathrm{~cm}$ and $25 \mathrm{~cm}$ in height, and its mean value was calculated after repeating the exercise for three times (Figure 1). After the subjects performed the push-up exercise under one condition and a 30-minute break was given in order to prevent them from accumulating fatigue before the next push-up exercise under the other condition.

\section{Data analysis}

The signals were processed in the following manners. Amplified waves were filtered through a Bandpass filter of 10-350 Hz. The mean value of root mean squares (RMS) was used for each muscle's activity. SPSS 20.0 for windows (IBM Corporation, USA) was used for comparative analysis of the measured data in the present study. The collected data was analyzed using an independent t-test, and statistical significance was set at $\mathrm{p}<0.05$.

\section{RESULTS}

\section{General characteristics}

The mean age of the study subjects was $24.14 \pm 0.53$ years old (23-25 years) with the average height of $174.43 \pm 5.24 \mathrm{~cm}(168-187 \mathrm{~cm})$ and the average weight of $71.43 \pm 8.68 \mathrm{~kg}(62-90 \mathrm{~kg})$.

\section{Difference in muscle activities of upper extremity muscles}

The study subjects showed lower muscle activities of the triceps brachii, serratus anterior and latissimus dorsi during the push-up exercise on a high and unstable support surface than on a stable support surface by $3.34 \%, 3.70 \%$, and $1.90 \%$, respectively, however, there were no statistically significant differences $(p>0.05)$.

Table 1. Difference of muscle activation in upper extremity muscle according to the height of lower extremity and condition of support surface

\begin{tabular}{clccc}
\hline & \multicolumn{3}{c}{ Upper extremity muscle } \\
\cline { 3 - 5 } & & Triceps brachiii & Serratus anterior & Latissimus dorsi \\
\hline High & Unstable & $22.05 \%(8.07)$ & $30.19 \%(11.46)$ & $15.43 \%(7.76)$ \\
& Stable & $25.39 \%(9.96)$ & $33.89 \%(10.93)$ & $17.33 \%(9.3)$ \\
& p-value & 0.338 & 0.389 & 0.562 \\
\multirow{2}{*}{ Low } & Unstable & $24.96 \%(8.26)$ & $38.36 \%(10.15)$ & $16.34 \%(9.56)$ \\
& Stable & $21.65 \%(10.17)$ & $27.86 \%(9.6)$ & $14.11 \%(5.99)$ \\
& p-value & 0.353 & 0.116 & 0.467 \\
\hline
\end{tabular}

Values represent mean (standard deviation). 
Table 2. Difference of muscle activation in core muscle according to the height of lower extremity and condition of support surface

\begin{tabular}{clccc}
\hline & & \multicolumn{3}{c}{ Core muscle } \\
\cline { 3 - 5 } & & Rectus abdominis & $\begin{array}{c}\text { Abdominal } \\
\text { external oblique }\end{array}$ & Erector spine \\
\hline High & Unstable & $21.11 \%(11.07)$ & $19.86 \%(8.87)$ & $3.54 \%(1.34)$ \\
& Stable & $15.64 \%(9.00)$ & $17.51 \%(8.90)$ & $5.04 \%(3.06)$ \\
& $p$-value & 0.164 & 0.49 & 0.338 \\
\multirow{2}{*}{ Low } & Unstable & $22.31 \%(11.74)$ & $23.57 \%(10.17)$ & $4.91 \%(1.94)$ \\
& Stable & $13.06 \%(8.24)$ & $16.05 \%(7.56)$ & $4.14 \%(2.04)$ \\
& p-value & $0.023^{*}$ & $0.035^{*}$ & 0.319 \\
\hline
\end{tabular}

Values represent mean (standard deviation).

${ }^{*} p<0.05$.

On the other hand, the subjects showed higher muscle activities of the triceps brachii, serratus anterior and latissimus dorsi during the push-up exercise on a low and unstable support surface by $3.31 \%, 10.50 \%$, and $2.23 \%$, respectively, but there were no statistically significant differences $(\mathrm{p}>0.05)$ (Table 1$)$.

\section{Difference in muscle activities of core muscles}

The study subjects showed higher muscle activities of the rectus abdominis muscle and the abdominal external oblique muscle during the push-up exercise on a low and unstable support surface than on a stable support surface by $9.25 \%$ and $7.52 \%$, respectively, and there were statistically significant differences $(\mathrm{p}<0.05)$.

Nonetheless, there were no statistically significant differences in the erector spinea muscle $(\mathrm{p}>0.05)$. When the subjects performed the push-up exercise on a high and unstable support surface, the muscle activities of the rectus abdominis muscle, abdominal external oblique muscle and erector spinea muscle were either higher or lower by $5.47 \%, 2.37 \%$, and $-1.50 \%$ respectively, than on a stable support surface, however, there were no statistically significant differences $(\mathrm{p}>0.05)$ (Table 2).

\section{DISCUSSION}

The present study was conducted to investigate differences in muscle activities of the upper extremity muscles and the core muscles during push-up exercises on different support surfaces of the lower limbs. According to the results of the experiment, similar muscle activities were observed in both the upper extremity muscles and the core muscles during the push-up exercise on a high support surface of the lower limbs, whether the surface be stable or unstable.
However, on a low and unstable support surface of the lower limbs the push-up exercise appeared to induce a significant level of muscle activities of the abdominal muscles while it induced no particular differences in the muscle activities of the upper extremity muscles. It is thought that the push-up exercise on a low and unstable support surface leads to much more muscle activities of the abdominal muscles than the exercise on a high and unstable support surface.

The push-up exercise on a low and unstable support surface is expected to improve the muscle activities of not only the upper extremity muscles but also the core muscles, and enhances the exercise effects. From the previous studies of push-up exercise performed under various conditions, it has been known that the exercise can improve the strength of selective safe muscles in the shoulder joints with regard to changes in the length of the support surface. ${ }^{8}$ In addition, on top of the length of the support surface, the height and position of hands create differences of the muscle activities in the shoulders. ${ }^{19}$ Lear et al. ${ }^{10}$ reported that the push-up exercise using the combination of two different conditions enhanced the stability of the shoulder joint.

Moreover, exercises on an unstable surface have been proven to enhance the muscle activities of the upper extremity muscles through many studies. ${ }^{3,16}$ In addition, the exercises have been reported to induce not only more muscles activities but also more endurance of the muscles, and help improve coordination of muscles. ${ }^{21}$ In particular, exercises on an unstable surface have been known to maximize the exercise effects as they contract both the agonist muscle and the synergistic muscle simultaneously to induce improvement in the strength, balancing ability and stability of the muscles by stimulating the neurotransmitter system. ${ }^{19}$ In addition, Oh et al. ${ }^{22}$ reported that when a push-up exercise on a stable surface and a push-up exercise with a sling on an unstable surface were compared, the exercise on an unstable surface induced high muscle activities of the upper extremity muscles. However, a variety of the recent studies have reported on not only muscle activities of the upper extremity muscles during exercises on an unstable surface but also comparison of different patterns in muscle activities of the core muscles, which are related to the trunk..$^{13,14,23}$

In terms of core muscle, Petrofsky et al. ${ }^{13}$ reported that different core muscle activation during abdominal crunches between stable surface and unstable surface using Swiss ball. They suggested that the exercise on an unstable surfacecan induce higher muscle activi- 
ties of the core muscles, compared with those of stable surface. Furthermore, according to Escamilla et al., ${ }^{14}$ an abdominal exercise on a Swiss ball improved muscle activities of the core muscles in comparison to muscle activities induced during push-up and sit-up exercises on a Swiss ball. ${ }^{14}$ Considering the aforementioned findings of the previous studies in general, it is thought that they are in line with the findings of the present study, and moreover, exercises on an unstable surface can make the core muscles in the trunk more active.

In conclusion, through the present study, push-up exercise on a low and unstable surface of the lower limbs was shown to induce much higher muscle activities of the core muscles in the trunk. Such results are thought to suggest other effects of push-up exercise which was mainly used in improving the strength of the upper extremity muscles, and indicate that the exercise can be an effective exercise therapy to improve the strength of both the upper extremity muscles and the core muscles. The present study has limitations as it was confined to healthy male adults in their twenties, therefore, its results are inadequate to generalize to men and women of all age groups and patients. Hence, there is a need to broaden the test group to various age groups and women in future studies to investigate differences in muscle activities, and moreover, it is thought that additional studies are called for to analyze muscles activities during push-up exercise on a support surface of the knees among the elderly and infirm and women who otherwise, have difficulty to perform a typical push-up exercise. Then, the findings of such future studies can be used as data to develop exercise therapies to treat patients and other exercise programs.

\section{ACKNOWLEDGEMENTS}

“This research was supported by Basic Science Research Program through the National Research Foundation of Korea (NRF) funded by the Ministry of Education, Science and Technology (NRF2015R1D1A1A01060314)".

\section{REFERENCES}

1. Panjabi MM. Clinical spinal instability and low back pain. J Electromyogr Kinesiol. 2003;13(4):371-9.

2. Park Sk, Park JM, Lee JH. Effects of a push-up plus exercise program on scapular position and muscle activity in individuals with rounded shoulder posture. J Kor Phys Ther 2010;22(5):1-8.
3. Park SK, Lee OH, Kim JS et al. A comparison of muscle activity in periscapular muscles during push-up plus exercise on stable support and unstable support. The Journal of Korean Academy of Orthopedic Manual Therapy. 2005;11(2):71-82.

4. Ellenbecker TS, Davies GJ. Closed kinetic chain exercise: a comprehensive guide to multiple joint exercise. Champaign, Human Kinetics, 2001: 53-8.

5. Youdas JW, Budach BD, Ellerbusch JV et al. Comparison of muscle-activation patterns during the conventional push-up and perfect pushup exercises. J Strength Cond Res. 2010 ;24(12):3352-62.

6. Kim EY, Park HG, Ahn BH. Comparative studies of muscle activity on upper extremity between push-up bend and push-up plus movement according to change of supporting base interval. The Journal of Korean Academy of Physical Therapist. 2008;15(3):31-41.

7. Shim YH, Nam KS, Park JW. Effect of glenohumeral joint rotation on serratus anterior, pectoralis major and upper trapezius EMG activity during push-up plus exercise. J Kor Phys Ther. 2016;28(2):106-111

8. Anderson KG, Behm DG. Maintenance of EMG activity and loss of force output with instability. J Strength Cond Res. 2004;18(3):637-40.

9. Lehman GJ, MacMillan B, MacIntyre I et al. Shoulder muscle EMG activity during push up variations on and off a Swiss ball. Dyn Med. 2006;5:7.

10. Lear LJ, Gross MT. An electromyographical analysis of the scapular stabilizing synergists during a push-up progression. J Orthop Sports Phys Ther. 1998;28(3):146-57.

11. Cho YH, Kim SO, Choi JH. The differenes of shoulder muscle activity onset time according to body tilting angle in push-up exercise. J Korean Soc Phys Med. 2015;10(2):55-61.

12. Moseley JB Jr, Jobe FW, Pink M et al. EMG analysis of the scapular muscles during a shoulder rehabilitation program. Am J Sports Med. 1992; 20(2):128-34.

13. Petrofsky JS, Batt J, Davis N et al. Core muscle activity during exercise on a mini stability ball compared with abdominal crunches on the floor and on a swiss ball. Journal of Applied Research. 2007;7(3):255-72.

14. Escamilla RF, Lewis C, Bell D. Core muscle activation during Swiss ball and traditional abdominal exercises. J Orthop Sports Phys Ther. 2010; 40(5):265-76.

15. McGill SM, Grenier S, Kavcic N et al. Coordination of muscle activity to assure stability of the lumbar spine. J Electromyogr Kinesiol. 2003; 13(4):353-9.

16. Yoon JG. Relationship between muscle activity and kinematic variables of the upper extremity during a push up task on stable and unstable surfaces. J Kor Phys Ther. 2011;23(2):7-15.

17. Dolan P, Adams MA. The relationship between EMG activityand extensor moment generation in the erector spinaemuscles during bending and lifting activities. J Biomech. 1993;26(4-5):513-22

18. Lee GW, Yoon TL, Kim KS et al. EMG activity of abdominal muscles during lumbopelvic stabilization exercises. Korean Research Society of Physical Therapy. 2014;21(2):1-7.

19. Kim YS, Kim DY, Ha MS. Effect of the push-up exercise at different palmar width on muscle activities. J Phys Ther Sci. 2016;28(2):446-9.

20. Jung HK. The effects of the shoulder stabilizer muscle activity during a push up plus exercise according to presence or absence of visual information on stable and unstable support. Daegu University. Dissertation 


\section{TKPT $\begin{aligned} & \text { The Journal of } \\ & \text { Korean Physical Therapy }\end{aligned}$}

of Master's Degree. 2015.

21. Choi WI. An analysis of core muscle acitivity differences by knee joint angles in squat exercise on unstable surfaces. Korea National Sport University. Dissertation of Master's Degree. 2015.

22. Joaquin C, Sebastien B, Juan CC et al. Muscle activation differences be-
Sun-Uk Kim, et al.

tween stable push-ups and push-ups with a unilateral v-shaped suspension system at different heights. J Sports Sci Med. 2014;13(3):502-10.

23. Lee JS. Comparison of core muscle activity on surface conditions and loads during squat exercise. Dankook University. Dissertation of Master' s Degree. 2013. 